\title{
LORAWAN SCALABILITY ANALYSIS - CO SPREADING FACTOR INTERFERENCE
}

\author{
Aiju Thomas and N V Eldhose \\ School of Technology and Applied Science, M G University, \\ Edappally Kochi, Kerala, India
}

\begin{abstract}
Low Power Wide Area Networks (LPWAN) is on the verge of commercialization. The success of LPWAN technologies lies in the robustness of the modulation scheme. LoRa is a significant protocol in the segment which uses Chirp Spread Spectrum (CSS) as the modulation scheme. CSS is proven to be robust, ultra-low power consuming and resilient to noise and Doppler effects. Though noise resilience is profound, we investigate issues that may arise as the network scale. Co-Spreading Factor (Co-SF) interference is identified as a major issue that limits the performance of the network. Co-SF interference eventuates when multiple nodes trying to uplink simultaneously at the same Spreading Factor (SF) and almost at the near transmitted power level. Co-SF interference leads to packet error and escalation in the packet on air time which results in performance deterioration. Findings are justified through simulation and experimentation. Possible reasons leading to interference are constringed. Recommendations to reduce the effect of Co-SF interference are suggested and validated through experimentation.
\end{abstract}

\section{KEYWORDS}

LPWAN, Scalability, LoRa, LoRaWAN, co-spreading factor, Co-SF

\section{INTRODUCTION}

Recent years had shown unprecedented significance in Low power Wide Area Networks (LPWANs). Though cellular networks offer the highest portion of wireless connectivity, Internetof-Things (IoT) pave the way to a new segment of communication which characterizes low data rate but with significant coverage at low power. The niche solutions offered include LoRaWAN, Sigfox, Weightless, Telensa, Ingenu, Accellus, Cynet, Starfish, etc[1]. New standards IEEE 802.15.4k, NB-IoT, and LTE-M are recently adopted in the segment. Technologies like 4G/5G and WiFi access point offers high bandwidth connectivity catering requirements of transferring data at the high data rate. These are supplemented by Wireless Personal Area Networks (WPANs) [2], which transfer data to connected things at a high data rate but with limited coverage. WPAN is a low-powered, short-distance wireless network technology with a reach of few centimetres to a few meters. Bluetooth, ZigBee, WiFi, Wireless SB or IrDA are WPAN technologies operating at the ISM band following IEEE 802.15 standard [3].

The requirement of LPWANs to achieve energy efficiency and reaching longer distances are proven to be a challenge. LPWANs compromise data rates for range. The significance of LPWANs lies in the volume of IoT applications where a narrow band of data needs to be transmitted to the long-range. Most LPWANs operate in the unlicensed ISM bands at 169, 433, $868 / 915 \mathrm{MHz}$, and $2.4 \mathrm{GHz}$ depending on the region of operation [4]. There is significant research going on this end to evaluate and enhance the performance of low power networks. LoRaWAN is an emerging LPWAN for narrowband IoT applications. LoRaWAN is an attempt to deploy cost-effective solutions for autonomous applications. Cost-effectiveness implies a low cost of the transceiver, infrastructure, and operation. The paper analyzes scalability issues of LoRaWAN and suggests steps to enhance scalability. 


\section{Lora Physical Layer}

LoRaWAN is based on LoRA ${ }^{\mathrm{TM}}$ a patented technology by Semtech [5]. LoRa uses Chirp Spread Spectrum (CSS) for its physical layer. Chirps are linearly varying frequency carriers in the range $\left[f_{l}, f_{h}\right]$ forming up chirps or $\left[f_{h}, f_{l}\right]$ forming down chirps for a symbol time T, [6] where $f_{l}$ is the lower frequency and $f_{h}$ the higher frequency in the spectrum. Each symbol is modulated for the entire band width $B W=\left(f_{h}-f_{l}\right)$ thus spreading the entire spectrum. Employing $\mathrm{M}$ - Array modulation scheme [6], symbols are modulated as circular shifts of up chirps, the shifts characterizing each symbol are formed by multiple chips of duration $T_{c}=\frac{1}{B W}$. The modulating symbol of the $\mathrm{m}^{\text {th }}$ symbol can be represented as

$$
f(t)=\left\{\begin{array}{cl}
f_{h}+\eta\left(t-m_{c}\right) & \text { for } 0 \leq t \leq \mathrm{mT}_{c} \\
f_{l}+\eta\left(t-\mathrm{mT}_{c}\right) & \text { for } \mathrm{mT}_{\mathrm{c}} \leq \mathrm{t} \leq T_{s m}
\end{array}\right.
$$

Where $T_{s m}$ is the total symbol duration and the slope of linear frequency variation is represented as $\eta=\frac{\left(f_{h}-f_{l}\right)}{T_{s m}}$.

Within the given the bandwidth BW, the total number of symbols that can be encoded in CSS is given as $2^{n}$ where $n$ is the Spreading factor (SF) and $T_{c}$ the chip duration, the symbol duration $T_{s m}$ requires for encoding $2^{n}$ isgiven as

$$
T_{s m}=2^{n} T_{c}=\frac{2^{S F}}{B W}
$$

From the equation it is clear that for high SF time on-air is proportionally higher [5]. For LoRa transmission, $S F \in\{6,7,8,9,10,11,12\}$.

\subsection{Sensitivity of Receiver}

The sensitivity of LoRa device S at ambient temperature is given as [7]

$$
\mathrm{S}=-174+10 \log _{10} B W+N F+S N R
$$

The first term -174 is the effect of thermal noise in $1 \mathrm{~Hz}$ of bandwidth and is influenced by the receiver temperature. NF is the noise figure which is fixed for the hardware implemented. BW and SNR are the design criteria.

\section{LORAWAN}

LoRaWAN is a Media Access Control (MAC) protocol mapped to the second and third layer of the OSI model standardized by LoRA Alliance. Operating at sub $\mathrm{GHz}$ band maximum transmit power is limited to $21 \mathrm{dBM}$ and $14 \mathrm{dBm}$ for the US and Europe respectively [8]. LoRaWAN specifies three classes of devices depending on the uplink and downlink. 


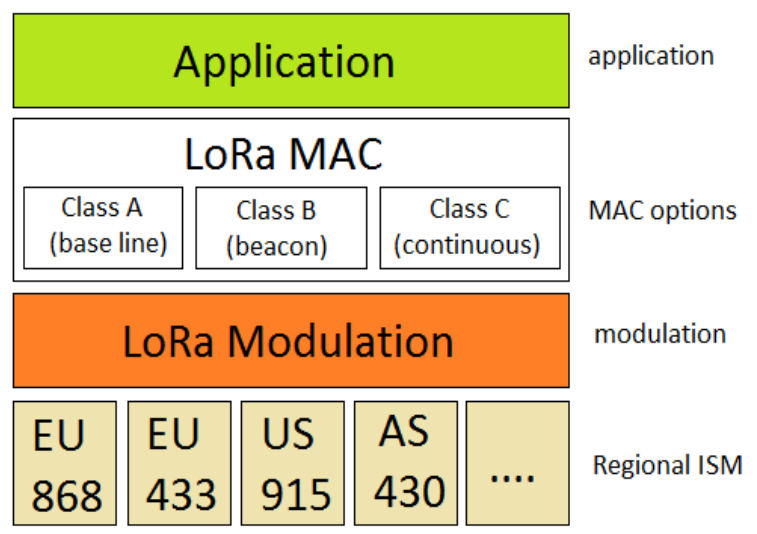

Figure 1.LoRaWANclasses

\subsection{LoRa WAN devices}

Class - A devices [8] are bi-directional where transmission slots are scheduled in random whenever a communication is needed based on pure ALOHA. The devices are in sleep mode unless there is something to transmit saving power. Uplink messages send by end devices are received and relayed by one or more gateways in the vicinity to the network server. It is only after an uplink by the device that the LoRa server can initiate a downlink. The server de-duplicates frames received by multiple gateways. Received frames are encrypted by the MAC-layer and payload is sent to the application server [7]. MAC layer prepares a downlink queue on which the application layer enqueue payloads for downlink. First downlink payload is transmitted on a subsequent receive window. The MAC layer acknowledges the application server as soon as the payload is acknowledged by the device. If an uplink is not acknowledged, a negative acknowledgment (nACK) is sent to the application server. Once the device is reactivated, the queue is flushed. Since Class A devices are in sleep mode unless there is something to transmit, they are the most preferred end devices configuration for LPWANs. Our investigation on scalability is based on Class A for experimentation.

Class B devices [8] are also supported by the LoRa Server. The internal clock of Class B devices is synchronized with beacons transmitted by the gateway called "beacon lock". LoRa server schedules downlink negotiating on the ping interval of the beacons. The server maintains all downloads for its devices in the queue. Whenever a device is beacon locked, the payloads are scheduled for the next free ping slot in the queue.

Class C devices [8] are an extended version of Class A. These devices keep receive windows open for all time except when they are transmitting. These devices are very much energyconsuming though they provide low latency communication.

\subsection{Frequency Band}

Operating at unlicensed band parameters specified for the region of operation is applicable. European Union imposes duty cycle restrictions for each sub-band in $863-870 \mathrm{MHz}$ ISM band at which LoRa operates. Since these restrictions are applied to every device, both gateway and devices are bound to follow duty cycle regulations. Most of the channels used by LoRaWAN in the region have duty cycle restrictions from $0.1 \%$ to $1 \%$. Applications using LoRaWAN have to keep payloads small and have to keep download to as much minimum as possible. 
LoRa uses the $902-928 \mathrm{MHz}$ band in the United States (US). Unlike the European Union, the US permits separate uplink and downlink channels in the band. The entire ISM band is divided into eight sub-bands in the region, each with eight $125 \mathrm{kHz}$ uplink channels, one $500 \mathrm{kHz}$ uplink channel and one $500 \mathrm{kHz}$ downlink channel. The LoRa network uses the second sub-band for communication in the region.

China specifies $779-787 \mathrm{MHz}$ and $470-510 \mathrm{MHz}$ band for ISM operations in the sub-Giga $\mathrm{Hz}$ range. The $779-787 \mathrm{MHz}$ band has similar behavior to European Union in the region. The band had three $125 \mathrm{kHz}$ channels in the frequency $779.5 \mathrm{kHz}, 779.7 \mathrm{kHz}$, and $779.9 \mathrm{kHz}$. The behavior of $470-510 \mathrm{MHz}$ band is similar to that of the US. This band specifies 96 uplink and 48 downlink channels. Few subsets are being used by Chinese Power Company which restricts usage for ISM. LoRaWAN uses the eleventh sub-band for operation in China.

The Australian usage specifications for the $918-928 \mathrm{MHz}$ ISM band are the same as that of the US. The downlink channels use the same frequency range as with the US at $902-928 \mathrm{MHz}$ Uplink uses frequency from 915 - 902. The IoT network uses the second sub-band in the range. LoRaWAN uses $125 \mathrm{kHz}, 250 \mathrm{kHz}$ or $500 \mathrm{kHz}$ bandwidth depending on the region of operation. Usage restrictions in additions to investigated factors can also limit scalability. Such factors are not considered in this study. Spectrum utilization adds to regional incompatibility.

\subsection{Addressing}

LoRaWAN provides an identifier for device or application. The following are the address associated with any device which is logged into the network.

- Device unique hardware ID (DevEUI) is a 64-bit unique address similar to the MAC id of TCP/IP.

- Device unique hardware ID (DevAddr) is a 32-bit non-unique dynamic address provided or chosen when the device joins a network.

- Application ID (AppEUI) is an Extended Unique Identifier (EUI) - 64 address is a unique ID provided by the application. AppEUI is stored in the end-device before application activation.

- End applications or services are identified by Fport. MAC messages reserve usage of Port 0 . Fport is compared with TCP/UDP port number of TCP/IP devices.

The addressing information is included as Physical Header (PHY_Header) which adds to the payload of LoRa. An explicit mode of operation adds header information. The implicit mode avoids header information and has to be configured manually. The implicit mode of operation reduces packet error time but cannot be used for a network with multiple end devices.

\subsection{Adaptive Data Rate}

LoRa changes the data rate and transmits power as a function of signal quality and distance. Dynamic selection of SF compromise data rate. At higher SF, packet time on-air is higher compared to lower values, as the signal quality reduces and distance increases compromise data rate. 


\subsection{Data Redundancy}

LoRa uses forward error correction (FEC) to recover data bits corrupted by interference. The additional overhead of encoding is added to data for redundancy. Using hamming code at code redundancy (CR) 4/5, 4/6, 4/7, 4/8 synonymies as CR 1, 2, 3 and 4 increases time on air for the same spread factor. CR is selected based on the channel condition. Adding coding bits to data, $R_{b}$ the bit rate is

$$
R_{b}=S F \frac{\left[\frac{4}{4+C R}\right]}{\left[\frac{2 S F}{B W}\right]}
$$

\subsection{Hardware}

Experimentation is performed in this study using the SX1276 series LoRa transceiver in a single antenna mode [8]. Semtech SX127X series allows single or double antenna configuration for transmitting and receive. Optimal sensitivity is achieved using separate antennas or with RF switching between Transmit (Tx) and Receive (Rx). Former provides better sensitivity to -131 $\mathrm{dBm}$ against $-128 \mathrm{dBm}$ for a single antenna version [14]. LoRaWAN uses simple ALOHA.

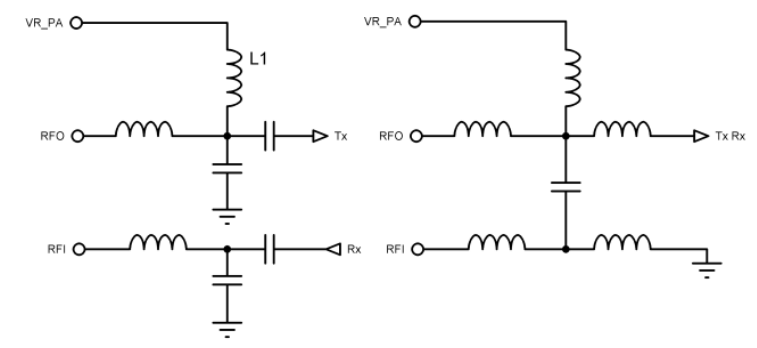

Figure2.Individual and shared TX Rx

\subsection{Factor affecting time on-air Class A transceivers}

LoRa transmissions are subjected to regulatory and system-level design constraints on time on-air and receiver sensitivity. When transmitted power had a direct relation with receiver sensitivity, time on-air depends on packet formatting. LoRA packet comprises "npreamble" symbols to mark the beginning of a packet, "nheader" symbols as node identifier with CR 4/8 and payload with selected CR. The packet format is shown in figure 3. Uplink messages are transmitted in explicit mode with physical header [10] (PHDR) with optional CRC or implicit mode without header information.The header uses CRC for data integrity and uses CRC4/8 by default. The radio transceiver inserts pay-load and CRC with the physical header. Hence time on air for packet transmission depends on mode selection as explicit or implicit, SF and CR applied for transmission. Downlink messages are sent to the end device through a single gate-way by the network server. These massages use explicit mode with CRC.

\begin{tabular}{|c|c|c|c|c|}
\hline \multirow{2}{*}{ Preamble } & \multicolumn{2}{|c|}{ (explicit mode) } & \multirow{2}{*}{ Payload } & \multirow{2}{*}{ CRC } \\
\hline & PHY_Header & PH_CRC & & \\
\hline
\end{tabular}

Figure 3.LoRa packet format 
The evaluation performed in this paper uses Class A device as a node for its power-saving feature. After every packet transmission, the end device waits for one of two receive slots which happen in a fixed interval, taking the end of transmission as reference. Figure 4 demonstrates Class A transmission. The RX1 slot uses frequency and data rate as a function of the uplink. RX1 is opened for receive delay 1 second with a tolerance of $\pm 20 \mu$ secs after the end of packet transmission. The delay and data rate are determined by specifications applicable to the region of operation. The second slot RX2 uses configurable fixed data rate and frequency.

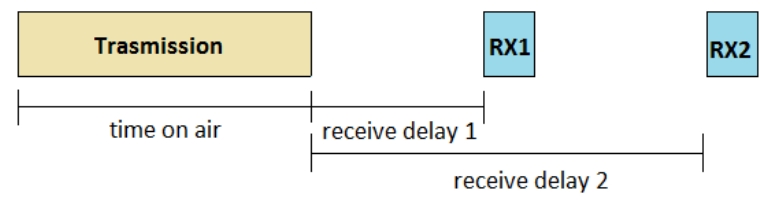

Figure 4.Class A device

The slot opens after a receive delay of 2 seconds with an error factor of $\pm 20 \mu$ secs. The receive window duration is determined by the time required by the end device radio to detect downlink preamble. If the preamble is detected during any receive slot, the end device radio remains active until the entire downlink frames are received and decoded. If data integrity is verified during the first receive slot, subsequent receive slot is not opened and the device goes on sleep. If acknowledgments are not received or decoded to the required integrity even after the second receive window, the uplink is scrapped for retransmission which can happen after the second transmit window of the previous session is expired. The device listens to other protocols operating in the frequency range before transmissions complying with the regulations prevailing in the region. Failed uplink or downlink along with regional specifications adds to the packet time on air. Interference of types to be discussed or poor SNR characteristics can contribute to erratic uplink or downlink. If the network server intends to do a downlink to the end device, it initiates transmission precisely at the beginning of any of the receiving slot. To calculate time on-air for a transmitted packet:

Given $T_{s m}$ as symbol duration in equation (2)

The time duration $T_{p h}$ for $n_{p h}$ preamble signal

$$
T_{p h}=\left(n_{p h}+4.25\right) T_{s m}
$$

The value 4.25 accounts for two up chirps, two down chirps and a quarter up chirp which add to preamble for synchronizing the clock which is otherwise called 'SynchWord'.

Number of symbols $N_{p l}$ contained in the packet that makes header and payload are given as

Where

$$
N_{p l}=8+\max \left(\operatorname{ceil}\left(\frac{8 P L-4 S F+28-20 H}{4(S F-2 D E)}\right)(C R+4), 0\right)
$$

- PL is the number of payload bytes.

- $\mathrm{H}=0$ for explicit mode or $\mathrm{H}=1$ for non implicit mode.

- $\mathrm{DE}=1$ when low data rate optimization is enabled or $\mathrm{DE}=0$ when disabled.

- $\mathrm{SF}$ is the spreading factor and CR is the coding rate (1 to 4 ).

The payload duration $T_{p l}$ is given as 
International Journal of Computer Networks \& Communications (IJCNC) Vol.12, No.1, January 2020

$$
T_{p l}=N_{p l} T_{\text {sym }}
$$

Time on-air or packet duration

$$
T_{\text {packet }}=T_{p h}+T_{p l}
$$

Table 1 shows the theoretical performance of LoRa for different SF for a payload of 8 bytes at $125 \mathrm{kHz}$ bandwidth and centre frequency $868 \mathrm{MHz}$, transmit power of $14 \mathrm{~dB}$ in the explicit mode for different CR calculated using equation 8 .

\begin{tabular}{|c|c|c|c|c|c|}
\hline SF & $\begin{array}{l}\text { Bit rate } \\
\mathrm{Kb} / \mathrm{s}\end{array}$ & $\begin{array}{l}\text { Packet } \\
\text { air time } \\
(\mathrm{m} \mathrm{sec})\end{array}$ & $\begin{array}{c}\text { Symbol } \\
\text { time } \\
(\mathrm{m} \mathrm{sec})\end{array}$ & $\begin{array}{c}\text { Receiver } \\
\text { sensitivity } \\
(\mathrm{dBm})\end{array}$ & $\begin{array}{l}\text { Link budget } \\
\text { (db) }\end{array}$ \\
\hline \multicolumn{6}{|c|}{$\mathrm{CR}=1(4 / 5)$} \\
\hline 7 & 5.480 & 36.10 & 1.02 & -120.0 & 134.0 \\
\hline 8 & 3.125 & 72.19 & 2.05 & -123.0 & 137.0 \\
\hline 9 & 1.725 & 123.90 & 4.10 & -126.0 & 140.0 \\
\hline 10 & 0.976 & 247.81 & 8.19 & -129.0 & 143.0 \\
\hline 11 & 0.537 & 495.62 & 16.38 & -131.5 & 145.5 \\
\hline 12 & 0.292 & 991.23 & 32.77 & -134.0 & 148.0 \\
\hline \multicolumn{6}{|c|}{$\mathrm{CR}=2(4 / 6)$} \\
\hline 7 & 4.557 & 39.17 & 1.02 & -120.0 & 134.0 \\
\hline 8 & 2.604 & 78.34 & 2.05 & -123.0 & 137.0 \\
\hline 9 & 1.468 & 132.1 & 4.10 & -126.0 & 140.0 \\
\hline 10 & 0.813 & 264.19 & 8.19 & -129.0 & 143.0 \\
\hline 11 & 0.447 & 528.38 & 16.38 & -131.5 & 145.5 \\
\hline $12 \mid$ & 0.244 & 1056.77 & 32.77 & -134.0 & 148.0 \\
\hline \multicolumn{6}{|c|}{$\mathrm{CR}=3(4 / 7)$} \\
\hline 7 & 3.966 & 42.24 & 1.02 & -120.0 & 134.0 \\
\hline 8 & 2.232 & 84.48 & 2.05 & -123.0 & 137.0 \\
\hline 9 & 1.255 & 140.29 & 4.10 & -126.0 & 140.0 \\
\hline 10 & 0.697 & 280.58 & 8.19 & -129.0 & 143.0 \\
\hline 11 & 0.383 & 561.15 & 16.38 & -131.5 & 145.5 \\
\hline 12 & 0.209 & 1122.30 & 32.77 & -134.0 & 148.0 \\
\hline \multicolumn{6}{|c|}{$\mathrm{CR}=4(4 / 8)$} \\
\hline 7 & 3.417 & 45.31 & 1.02 & -120.0 & 134.0 \\
\hline 8 & 1.953 & 90.62 & 2.05 & -123.0 & 137.0 \\
\hline 9 & 1.098 & 148.48 & 4.10 & -126.0 & 140.0 \\
\hline 10 & 0.610 & 296.96 & 8.19 & -129.0 & 143.0 \\
\hline 11 & 0.335 & 593.92 & 16.38 & -131.5 & 145.5 \\
\hline 12 & 0.183 & 1187.84 & 32.77 & -134.0 & 148.0 \\
\hline
\end{tabular}

Table 1. Time on air characteristics for 8 -bit data at $125 \mathrm{kHz}$

\section{RELATED WORK}

Bit Error Rate [BER] analysis of LoRa[9] shows excellent noise rejection characteristics of the modulation scheme. The demonstration in paper [9] shows significant variation from -20dB for $\mathrm{SF}=12$ against $-6 \mathrm{~dB}$ for $\mathrm{SF}=7$ proves less possibility of packet error due to poor SNR characteristics of the channel. Dynamic selections of SF based on channel characteristics improve the noise level performance of LoRaWAN. Analysis performed in paper [9] with 500 end devices transmitting in random SF for 60 seconds at $125 \mathrm{KHz}$ bandwidth shows packet collisions and 
shoes significantly higher packet error rate at higher SF compared to that at lower. This is in contrary to noise performance at higher SF. The paper raises concerns LoRaWAN scalability.

There are attempts [10] to model up linking to a single gateway taking into account signal of co spread factor interference using tools of stochastic geometry. The paper considers the spatial distribution of end devices which are uniformly located at random deployment region $A=\pi r^{2}$. The paper assumes that symbols modulated as up-chirps or down-chirps for each chunk of bits where in LoRa uses M array modulation of up chirps for symbols. The assumption made in the paper hold good for the mathematical model presented, analysis with the actual modulation scheme can be rather complicated. All devices transmit in simple ALOHA satisfying a 1\% duty cycle specified in ETSI[17]. To satisfy duty cycle norms, the device with higher SF transmit less often. The analysis is performed for BW $=125 \mathrm{kHz}$ with spatially located and uniformly random through inhomogeneous Poison Point Process (PPP). The paper observes received signals at the same frequency and SF interferes at gateway result in packet loss. Collisions cause retransmission inherent with ALOHA, wasting considerable power. Assumptions are made as SF is allotted by the Net server depending on the distance from the gateway. The model observed that chirp signals interfere among themselves and packets collide in time, frequency and spreading factor. Leveraging on tools available on stochastic geometry, two outrage conditions based on SNR and co-spreading sequence interference were demonstrated which through lights on scalability issues of LoRa. Though conditions limiting scalability is modelled mathematically, the paper suggests further analysis on the issue based on packet-level simulation tools.

The letter [6] analyse the link-level performance of LoRa due to imperfect orthogonality. Error due to imperfect orthogonality can result when signals with different spreading factors interfere or there is an error in synchronization while decoding the symbols. The letter highlights issues on scalability when multiple gateways are installed. The authors had developed LoRA physical layer simulator in MATLAB [13] which demonstrates link-level performance for different spreading factors. Simulations and experimentations performed with SX1271 transceiver demonstrate collisions among chirp signals at different SFs. The tabulation of signal to interference threshold suggests a high level of rejections due to collisions among signals with the same SF. The letter suggests that due to imperfect orthogonality among spreading factors, a LoRa network cannot be considered as simple super positioned independent networks. When the interference signals over powers the reference signal, the erratic behaviour of demodulation can result.

\section{SCAlAbILITy ANALYSIS OF LoRA}

\subsection{Packet error Loss}

The salient feature of LoRa which makes it sturdy as spread spectrum lies in orthogonality. They are orthogonal to any other signals including those which are spread in the same bandwidth. The receiver upon detecting preamble signals generates down chirps at the same SF which are synchronized by "SyncWord".

$$
\begin{gathered}
\mathrm{f}_{\mathrm{m}}=-\eta \mathrm{mT}_{\mathrm{c}} \\
\mathrm{f}_{\mathrm{m}}-\mathrm{BW}=-\left(\mathrm{f}_{\mathrm{h}}-\mathrm{f}_{\mathrm{l}}\right)-\eta \mathrm{mT}_{\mathrm{c}}
\end{gathered}
$$

These two frequencies are aliased to $\mathrm{f}_{\mathrm{m}}$ which correspond to $\mathrm{m}^{\text {th }}$ symbol. The symbol $\mathrm{m}$ is estimated from the peak corresponds to the Fast Fourier Transform (FFT) of the multiplied. 
To study the effect of interference leading to packet error we performed simulations in MATLAB [12]. Overlapping transmissions of 500 devices transmitting in random SFs 7 to 12 are simulated[9]. At BW of $125 \mathrm{kHz}$ operating at frequency 868.7 to $869.2 \mathrm{MHz}$, packet collisions were analyzed for 60 seconds and are shown in figure 5 .

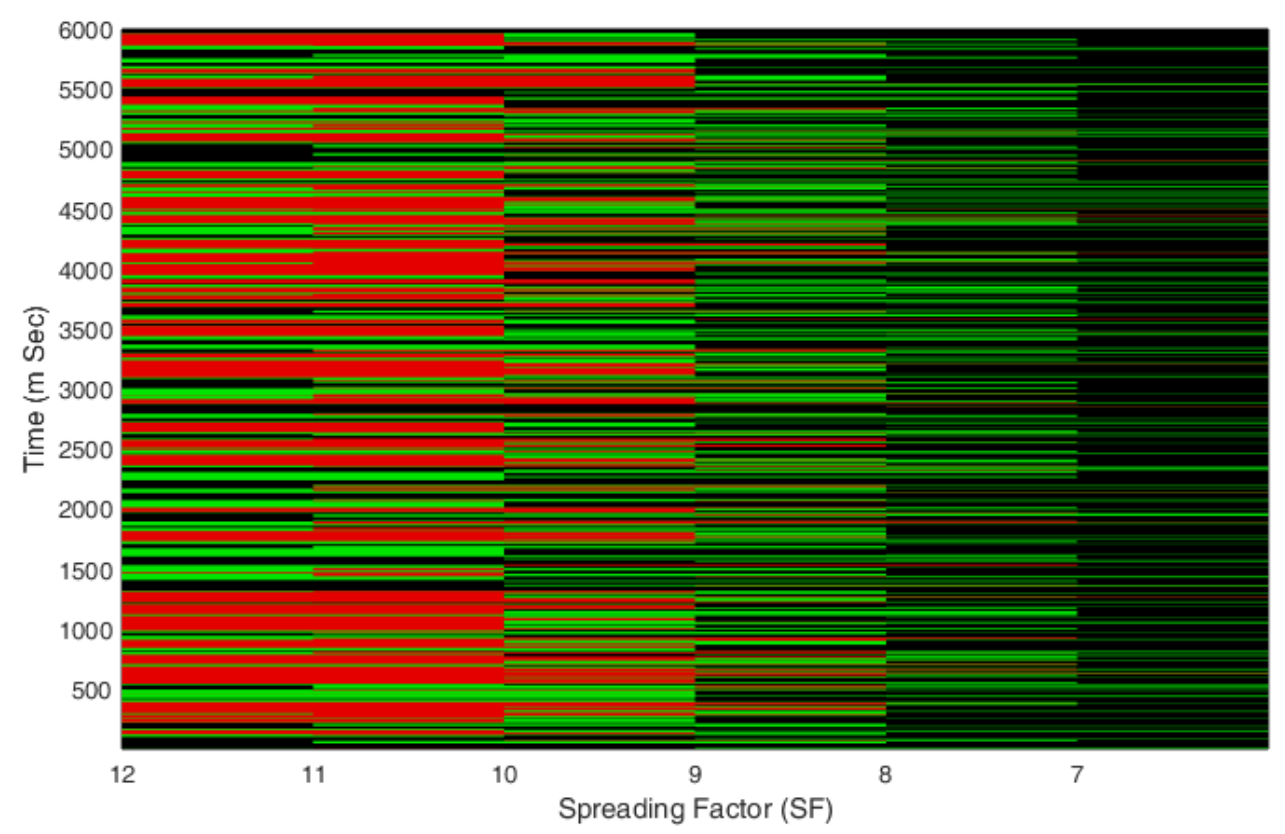

Figure 5. Collisions when 500 devices transmitting randomly at varying SF for 60 seconds at $125 \mathrm{KHz}$ bandwidth.

The plot reveals that at higher SF especially at 11 and 12, the collisions leading to packet error are surprisingly high. Though noise rejection performance at higher SFs and good, collisions can lead to retransmissions which increase time on air. This can degrade the performance of the network as it scales and can lead to violations of regional norms.

\subsection{Analysis of Packet outage}

The outage of packets can happen because of two conditions.

- Received SNR is below the threshold level required for error-free decoding. The level is $-20 \mathrm{~dB}$ for $\mathrm{SF}=12$ to $-6 \mathrm{~dB}$ for $\mathrm{SF}=7$ [11].This possibility is ruled out as the simulation is carried out applying the same SNR characteristics of an AWGN channel for all SFs.

- The other possibility is LoRa signals of different SFs interfering with one other creating packet loss.

Considering the second situation, the possibilities are constringed to two specific cases

1. Packets with different SFs interfering with creating inter SF interference.

2. Packets with same SFs colliding forming co-spreading factor interference.

The first case can be dismissed as signals of different SFs are quasi-orthogonal. Quasi-orthogonal signals cancel upon multiplication with a down chirp. Packet loss can happen only if the noise 
level is beyond the threshold and can hold very much for low SFs. On the contrary spectrogram in Figure 5 reveals thatcollisions are higher at higher SFs especially at 12 and 11 . The situation narrowed down to signals of the same SF interfering. Let us call this situation as Co-Spreading factor (Co-SF) interference where packets with the same SF interfere.

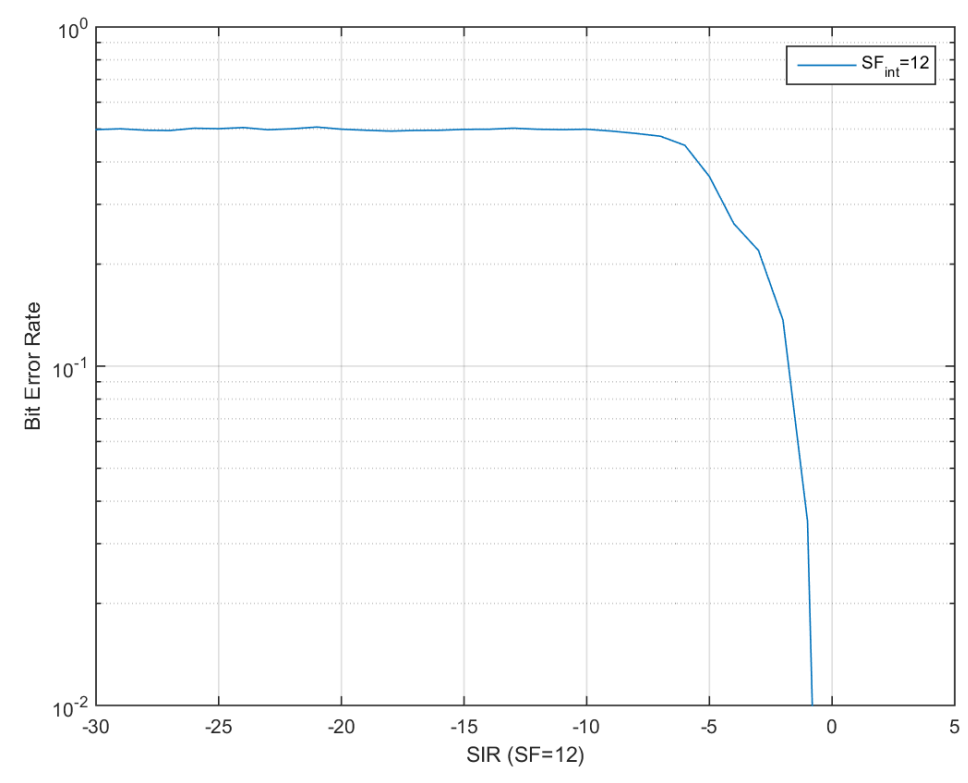

Figure 6.Bit Error Rate when packets of SF 12 interfering with 12

Co-SF interference happens when many end devices trying to uplink simultaneously. Since downlinks are synchronized by the net server and happen only during receive delay for class-A devices, the possibility Co-SF interference during downlink is a remote possibility. At higher SFs, the time on-air is longer and hence higher is the possibility of interference. Simulations were performed[13] on inter-SF interference when reference signals interfere with all interfering SFs from 6 to 12. The plot affirmed the fact that factor affecting scalability at SNR above the threshold is Co-SF interference.

Figures 6 and 7 show overlapping transmissions when SF 12 interfering with SF12 and SF 11. Figure 9 shows bit error performance when simulation repeated with SF 11which is interfered with signals from SF 6 to 12. For all interfering signals except that by SF11, the plot observes superior performance up to $-20 \mathrm{~dB}$ whereas when $\mathrm{SF}(\mathrm{SF}=11)$ interfere, performance degrades form $-1 \mathrm{~dB}$. The simulation is performed with packets that are transmitted simultaneously at CR $=3$ with interleaving and gray-coding as done in the LoRa physical layer [6]. Overlapping transmissions of reference signals and interfering signals are created by summing reference frames modulated at reference SF with those of interfering signals with interfering SF. Interfering packets are generated until the occurrence of 100 collisions, with a reference payload of 8 bytes.The plot demonstrates the Signal to Interference Ratio (SIR) threshold below which the packets are corrupted. Co- SF interference creates a low threshold level of SIR below which packet recovery is difficult due to high BER. Simulation confirms that Co-SF interference creates high BER leading to packet loss. 


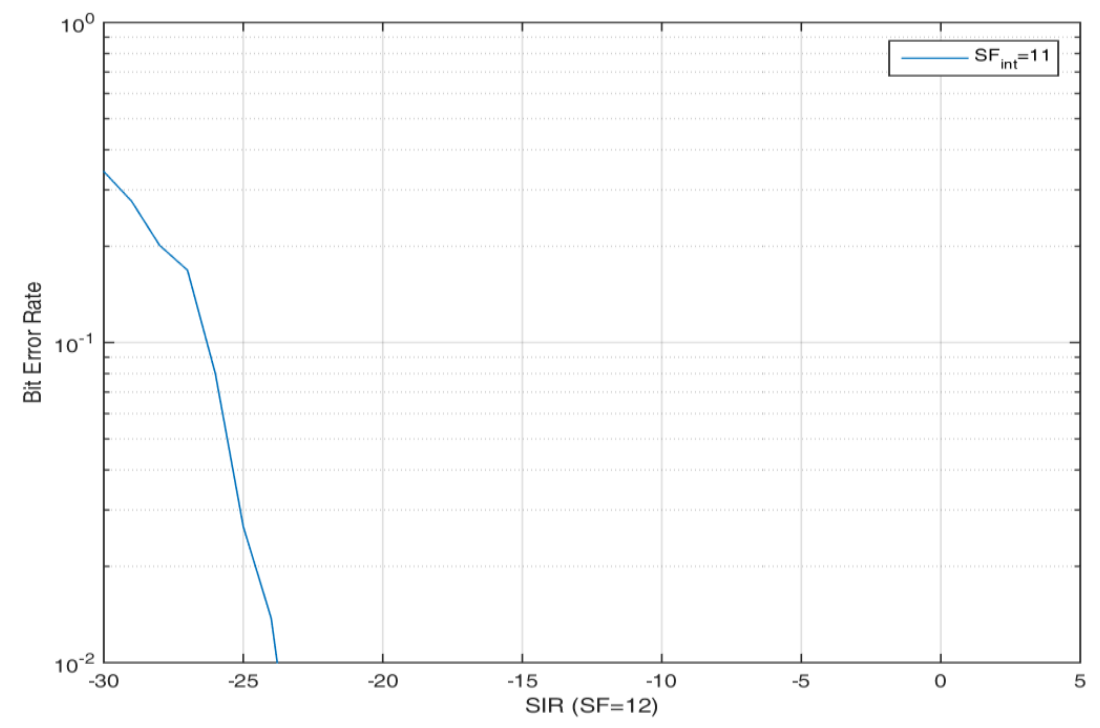

Figure7.Bit Error Rate when packets of SF 12 interfering with SF 11

LoRa employs several mechanisms as discussed before to make error recovery robust. This includes using Hamming coding and CRC [15]. Error packets can be reconstructed at the receiver side for correctness. Depending on the CR value, error decoding will be more efficient at higher $\mathrm{CR}$. But higher $\mathrm{CR}$ adds more burdens to the packet by adding additional bits. If errors are beyond recovery, the packet is scrapped and retransmitted. Before retransmission, acknowledgment slots are awaited. Each failed transmission adds on to packet air time and/or packet error. With $\mathrm{CR}=1$, the hamming code uses $1 \mathrm{CR}$ bit. Such case packet errors are seldom identified.

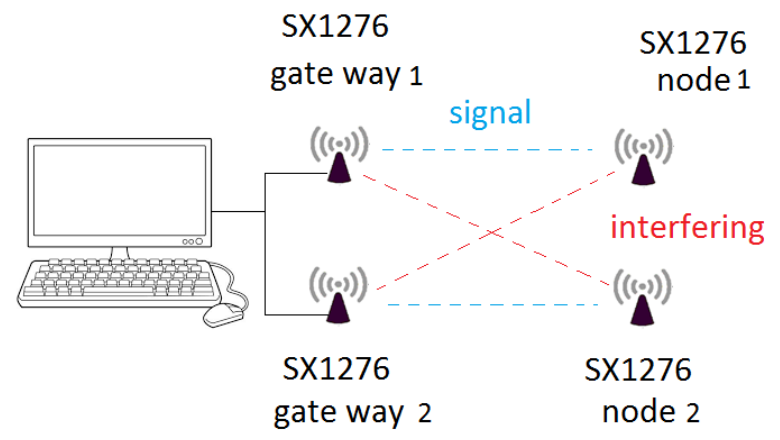

Figure 8.Experimental set up to study the effect of SF interference on packet air time 


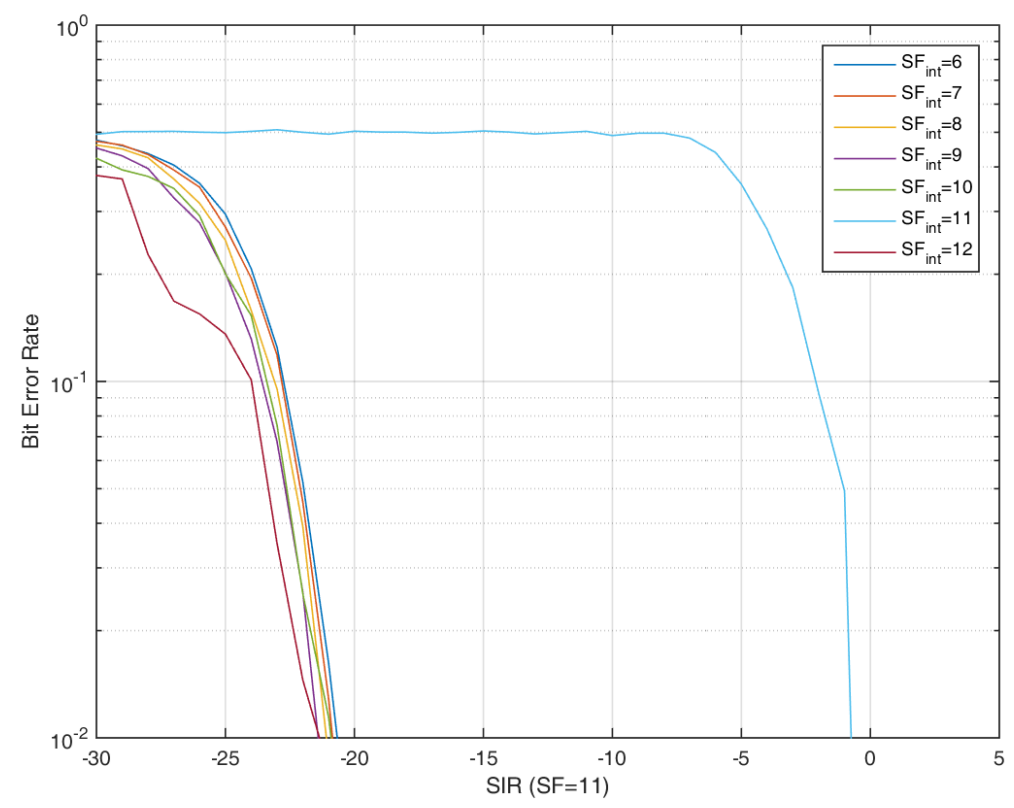

Figure 9.Bit Error Rate when packets of SF 11 interfering with SF 6 to 12

\subsection{Experimentation}

To characterize the behavior of LoRAWAN to Co-SF interferences, we have performed experimentation using LoRa SX1276. The experimental setup uses the LoRa SX1276 end-device per network connected to the SX1276 gateway. Two such networks were set as shown in figure 8.Network 1 uses gateways that are crafted using raspberry-pi, SX1276 hat and the end device (node) using SX1276,Arduino Uno. Data are logged and forwarded using the LoRa packet forwarder[16].Network2 similar to that of network 1sends data with random payload continuously. The two networks were implemented 50 meters in distance to maximize mutual interference and SNR. This also ensures that the threshold level of uplink signals and interfering signals are the same.

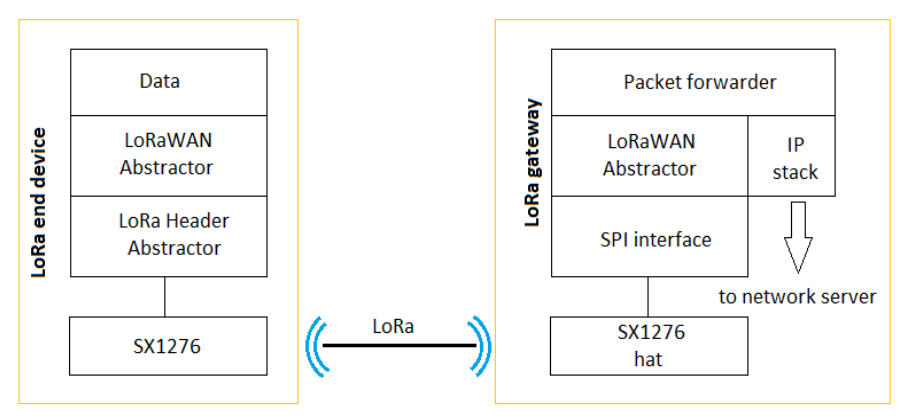

Figure10.Packet forwarder working on gateway

Eight bytes of a known payload is uplinked from node 1 to gateway 1 at a fixed interval. Packet transmission time on-air is calculated based on the known interval. The computational delay which gets added to the total time every time the data is sent is deducted. Data integrity is analyzed based on the payload. The computer system coordinates both networks so that the MATLAB code receives data and processes for possible conflicts. The software coordinates both 
networks, populates data on packet forwarder and implements corrective algorithms for testing the "Listen Before Talk" (LBT) algorithm. Table 2 shows parameters at which SX1276 transceivers are configured for node and gateway. The packet timing will vary if any of the parameters is reconfigured. Table 1 suggeststhe theoretical calculation for the mentioned criterion.

Table 2. Experimental Parameters

\begin{tabular}{|l|c|c|}
\hline \multicolumn{1}{|c|}{ Parameters } & Network 1 & Network 2 (interferer) \\
\hline SF & 7 to 12 & 7 to 12 \\
\hline Band width & $125 \mathrm{KHz}$ & $125 \mathrm{KHz}$ \\
\hline Preamble & 8 & 8 \\
\hline Coding rate & $1,2,3,4$ & $1,2,3,4$ \\
\hline Header mode & implicit & implicit \\
\hline Header CRC & disabled & disabled \\
\hline Pay Load CRC & enabled & disabled \\
\hline Hardware & RFIO is shared & RFIO is shared \\
\hline Pay load & 8 byte & 255 bytes \\
\hline Transmit power & $28.61 \mathrm{db}$ & $28.61 \mathrm{db}$ \\
\hline Center Frequency & $868 \mathrm{MHz}$ & $868 \mathrm{MHz}$ \\
\hline
\end{tabular}

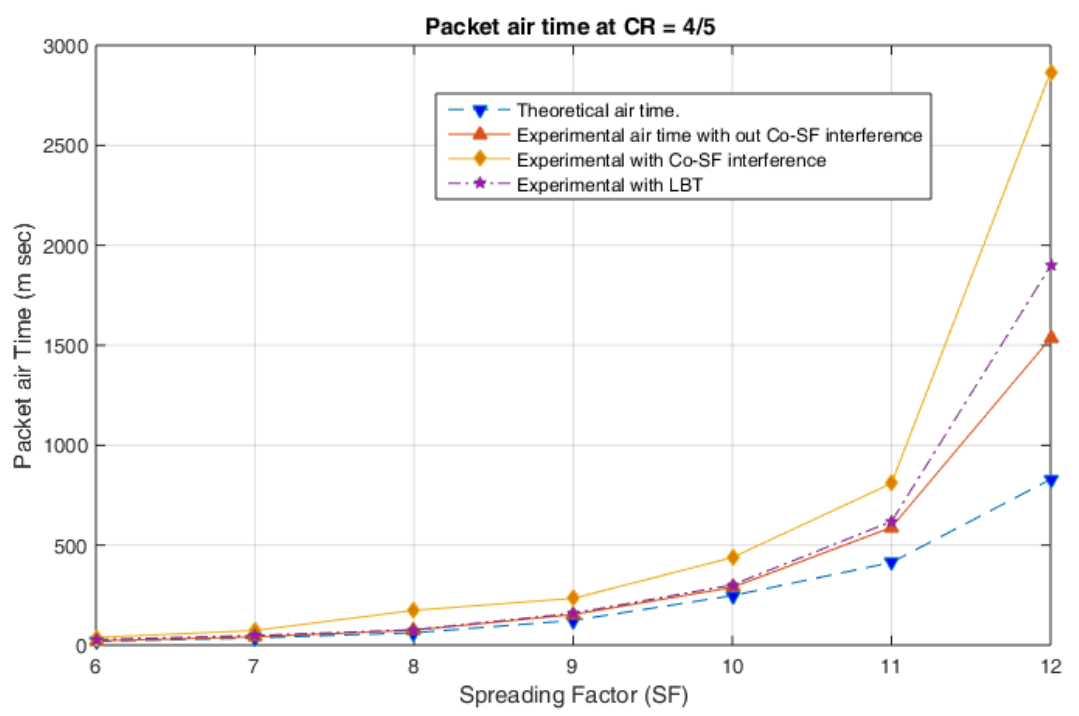

Figure11.Packet air time at $\mathrm{CR}=4 / 5$

At lower CR especially at 1,2 packet errors are significantly high in the presence of interference, while packet air time is lower. Whereas at $\mathrm{CR}=3$ and 4 , though lower packet error is observed, the packet on air time is significantly high. This is because at Hamming code 4/7 and 4/8 error correction properties are superior to that of $4 / 5$ and $4 / 6.4 / 5$ CRC is a simple parity check. If the packet is lost due to interference or any other reason, the gateway request for re-transmission during the acknowledgment cycle. This justifies the increase in packet air time as observed in the plot. The possibility of packet error to happen at higher CR is that the packet is totally lost or decoded erratically to match with CRC or the acknowledgment cycle is decoded erratically.

Along with error coding robustness of LoRa packet recovery is contributed by the whitening, shuffling, interleaving and gray coding [6] employed in LoRa. Gray coding maps adjacent bits in 
such a way that the bit pattern differs in a single position. LoRa shuffling and interleaving spread unreliable bits among several code words that even $4 / 5$ exhibit significant coding gain. Time on air calculation for the experimentation is made from an average of 10 experiments taken one after the other with a minimum possible delay. The minimum interval ensures that channel characteristics are not changed drastically due to other factors.

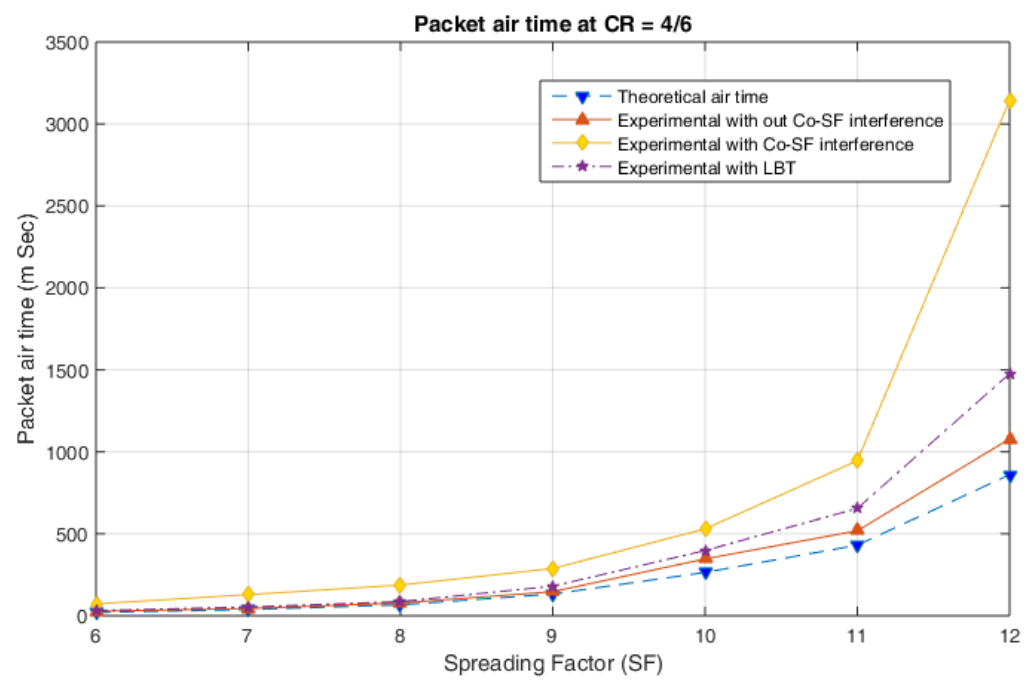

Figure12.Packet air time at $\mathrm{CR}=4 / 6$

SNR conditions and other channel characteristics are common in all cases of experimentation; the possibility of erratic decoding of packets or failed transmissions adding on to packet on air time can only be Co-SF interference. As the LoRa deployments scale, the chances of Co-SF interference multiplies much fold. Along with imperfections in orthogonality that can happen at the receiving end [5], when network scale in volume, packet time on-air drastically as shown. When errors are beyond recovery and the nodes attempt retransmission, channels get overloaded degrading the performance of the network. These justify concerns of network performance as LoRa networks scale. The possible reasons for Co-SF interferences limiting network scalability can be listed as:

- More LoRa network uses the same channel at the same SF and bandwidth for communication.

- There long queues of packets due to simultaneous transmissions resulting from uncoordinated node transmissions and pure ALOHA nature of the protocol.

- Failed transmissions add on to the packet queue.

- Uncoordinated gateways established by ad-hoc LoRa networks.

- Nodes communicating unbeknownst while gateways do downlink.

Delve into methods to reduce Co-SF interference, effectiveness of "Listen before talk" (LBT) algorithm is attempted. LBT examine carrier presence before data uplink. This method has a cumulative effect in reducing interference which ensures efficient channel utilization. 


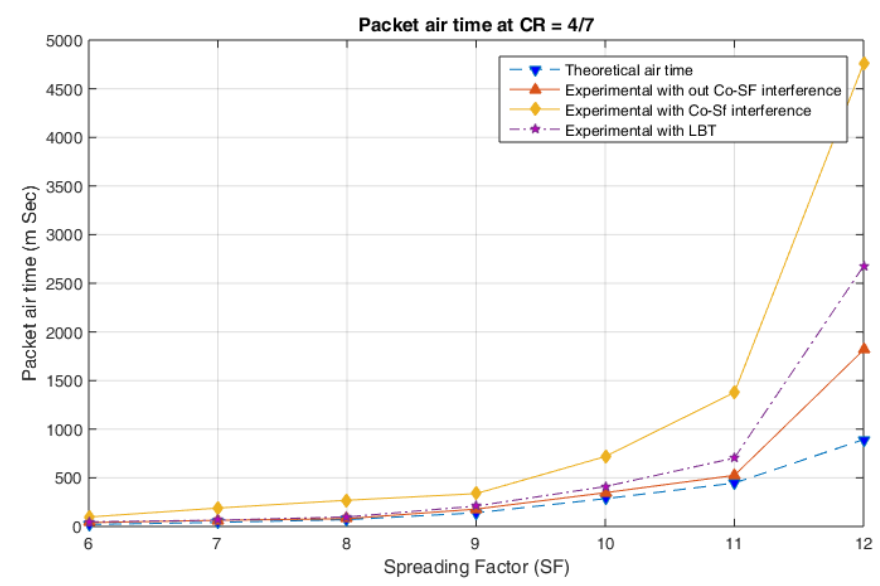

Figure13.Packet air time at $\mathrm{CR}=4 / 7$

To ascertain Co-SF interference and to prove the effectiveness of LBT, experiments were repeated for all the cases with LBT algorithms implemented through software. MATLAB codes check for any carrier that is present in the channel and coordinates both transmitting and interfering network so that only one network do the transmission. The plot reveals enhanced performance with the LBT algorithm implemented. A marginal increase in comparison with experimental values without interference accounts processing delay.

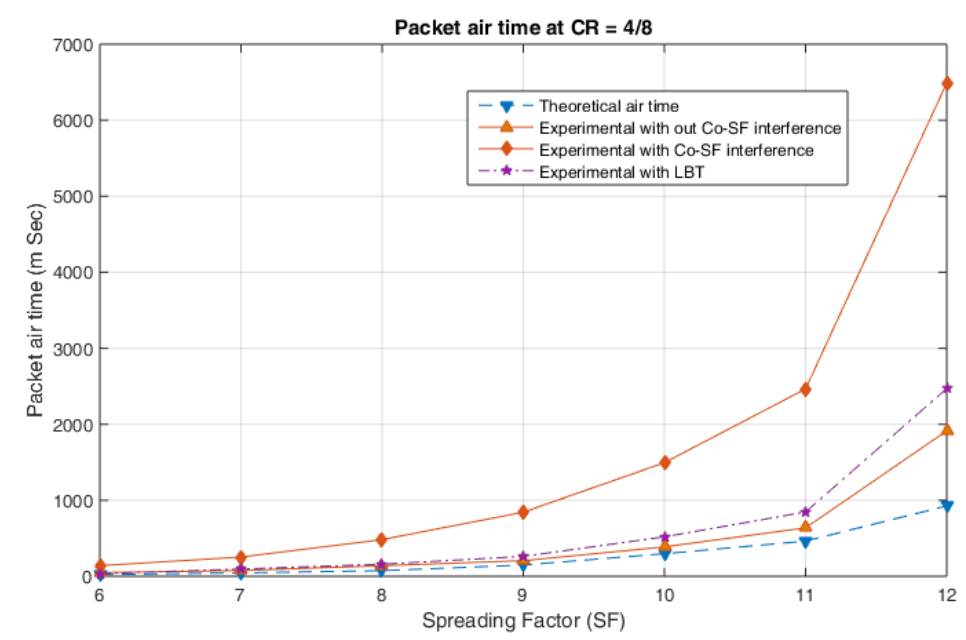

Figure14.Packet air time at $\mathrm{CR}=4 / 8$

\section{Conclusions}

We have analyzed through simulation and experimentation effect of Co-Spreading Factor interference leading to performance degradation of LoRaWAN. Imperfections in orthogonality results when LoRa networks use the same channel at the same spreading factor and bandwidth for communication. The performance analysis revealed that packet errors due to collisions are higher at higher SFs even though noise resilience characteristics are higher at these SFs. The study divulges that proneness to collisions at higher SFs is due to higher on air time. LoRa nodes communications are unbeknownst as uplinks are uncoordinated. The situation is aggrieved by long queues of retransmission due to the pure ALOHA nature of the protocol. Every failed transmission can be a burden to the network. Since the protocol operates at unlicensed band, adhoc or uncoordinated networks using the same or similar technology can worsen the situation. The situation can be improved by using slotted ALOHA. The probability of packet collisions can 
significantly low in this access control protocol. But the implementation needs the additional computational capability of the transceiver and has to keep the receiver on more time which in turn consumes additional power. Keeping the dynamic nature of SF and transmission power selection open for packet collisions can also improve the situation. Implementation of Quality of Service (QoS) can boost network performance. The paper suggests through experimentation that the LBT algorithm can enhance network performance at scaled conditions. LBT algorithm does not haul the system with additional computational complexity. "Listen Before Talk" is suggested as one of the solutionsto address scalability issues of LoRaWAN without compromising on computational or power restrictions of the protocol.

\section{REFERENCES}

[1] U. Raza, P. Kulkarni \& M. Sooriyabandara, (2017) "Low Power Wide Area Networks: An Overview", IEEE Communications Surveys \& Tutorials, Volume: 19, Issue: 2, pp855-873.

[2] R. Fisher, (2007) "60 GHz WPAN Standardization within IEEE 802.15.3c", IEEEInternational Symposium on Signals, Systems and Electronics, Volume: 1, pp103-105.

[3] A. Thomas \& N. V. Eldhose, (2015) "Driver encapsulation and transfer for machine to machine (M2M) communication", Proceedings of IEEE International Conference on Control Communication \& Computing India (ICCC), Vol.1, pp659-663.

[4] M Centenaro, L Vangelista, A Zanella \& M Zorzi, (2016) "Long-range communications in unlicensed bands: the rising stars in the IoT and smart city scenarios", IEEE Wireless Communications, Volume: 23, Issue: 5, pp60-67.

[5] Semtech, (2015) "LoRa Modulation Basics", AN1200.22 Semtech corporation ww.semtech.com, Revision 1, pp1-26.

[6] D. Croce, M. Gucciardo, S. Mangione, G. Santaromita \& I. Tinnirello, (2018) "Impact of LoRa Imperfect Orthogonality:Analysis of Link-level Performance", IEEE Communications Letters, Vol. 22, No. 4, pp796-799.

[7] Semtech (2013), “SX1272/3/6/7/8: LoRa Modem Designer's Guide”, AN1200.13Semtech corporation ww.semtech.com, Revision 1, pp1-9.

[8] N. Sornin \& A. Yegin, (2018) "LoRaWAN 1.0.3 Specifications," LoRa Alliance, Ver.1.0.3, pp 1-72.

[9] A. Thomas \& N. V. Eldhose, (2019) "Scalability Concerns of Chirp Spread Spectrum for LPWAN applications", International Journal of Ad hoc, Sensor \& Ubiquitous Computing, Vol. 10, No. 1, pp111.

[10] O. Georgiou \& U. Raza, (2017) “Low Power Wide Area Network Analysis: Can LoRa Scale?”,IEEE Wireless Communications Letters, Vol. 6, No. 2, pp162-165.

[11] A. Thomas \& N. V. Eldhose, (2019) "Performance Evaluation of Chirp Spread Spectrum as used in LoRa Physical", Proceedings of IEEE International Conference on Systems Computation Automation and Networking (ISCAN),Vol.1, pp214-219.

[12] "LoRaSim." www.lancaster.ac.uk/scc/sites/lora/ lorasim.html. online accessed October 2018.

[13] D. Croce, M. Gucciardo, S. Mangione, G. Santaromita \& I. Tinnirello (2018), "Simulation \& experimentation tools for LoRa networks", http:/lora.tti.unipa.it, online accessed November 2018.

[14] Semtech Corporation, (2016) “LoRa SX1276/77/78/79 Transceiver Datasheet”, www.mouser.com. Revision 5.

[15] J. Liando, A. Gamage, A. W. Tengourtius \& Moli (2018), "Known and Unknown Facts of LoRa: Experiences from a Large Scale Measurement Study" ACM Transactions on Sensor Network, Article 19. pp1-35.

[16] "Lora Packet forwarder",https://github.com/Lora-net/packet_forwarder, online accessed December 2018.

[17] D. Zucchetto\& A. Zanella, (2017)," Uncoordinated Access Schemes for the IoT: Approaches, Regulations, and Performance”, IEEE Communications Magazine, Volume. 55 , Issue. 9, pp48-54. 


\section{Authors}

Aiju Thomas received his Bachelor of Engineering and Masters of Technology in Electronics and Communication Engineering with specialization in microwave engineering. He is pursuing his research in the optimization of IoT protocols at M G University, Kottayam, Kerala, India. With 10 years of industrial experience and 19 years as faculty in Electronics and Communication Engineering at The Polytechnic Institute under State Government of Kerala, his field of interest includes industrial and embedded designing.

Dr. N V Eldhose received his Bachelor and Masters Degree in Science, Masters of Technology in Electronics and a Ph.D. degree in Physics. As a research guide for M G University, Kerala his field of interest includeubiquitous computing, wireless sensor networks, embedded systems, andmachine-to-machine communication. He is currently a faculty member in Electronics at the School of Technology and Applied Science, Kochi, India.
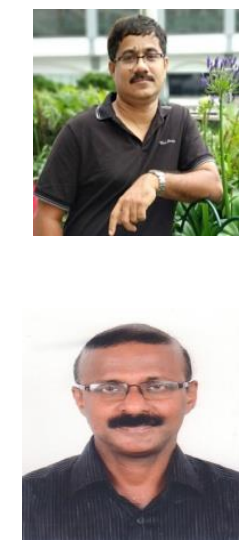